\title{
D-Glucosamine Conjugation Accelerates the Labeling Efficiency of Quantum Dots in Osteoblastic Cells
}

\author{
Kazunari Igawa, ${ }^{1}$ Ming-Fang Xie, ${ }^{2}$ Hideki Ohba, ${ }^{3}$ Shizuka Yamada, \\ and Yoshihiko Hayashi ${ }^{1}$ \\ ${ }^{1}$ Department of Cariology, Nagasaki University Graduate School of Biomedical Sciences, Nagasaki 852-8588, Japan \\ ${ }^{2}$ Biotechnology Division of Care Four Company Ltd., Kokura-Kitaku, Kitakyushu 802-0071, Japan \\ ${ }^{3}$ Measurement Solution Research Center, National Institute of Advanced Industrial Science and Technology, \\ Tosu, Saga 841-0052, Japan
}

Correspondence should be addressed to Yoshihiko Hayashi; hayashi@nagasaki-u.ac.jp

Received 26 November 2013; Accepted 3 March 2014; Published 24 March 2014

Academic Editor: Hideo Kusaoke

Copyright (C) 2014 Kazunari Igawa et al. This is an open access article distributed under the Creative Commons Attribution License, which permits unrestricted use, distribution, and reproduction in any medium, provided the original work is properly cited.

\begin{abstract}
Quantum dots (QDs) are useful imaging tools in the medical and biological fields due to their optical properties, such as a high fluorescence intensity, remarkable resistance to photobleaching, broad absorption spectra, and narrow emission spectra. This is the first study to investigate the uptake of carboxylated QDs conjugated with D-glucosamine (core size: approximately $3 \mathrm{~nm}$, final modified size: $20-30 \mathrm{~nm}$ ) into cultured osteoblastic cells. The QDs attached to the cell surface and were transported into the cytoplasm within approximately three hours of culture, whose process was clearly demonstrated using specific fluorescent staining of the cell membrane. Although the intranuclear distribution was not observed, a dramatic decrease in the transfer of quantum dots into the cytoplasm was recognized after approximately seven days of culture. Other interesting phenomena include the escape of the quantum dots from lysosomes in the cytoplasm, as confirmed by the merging of both QD fluorescence and specific fluorescent staining of lysosomes in the cytoplasm. These findings suggest that D-glucosamine conjugation enhances proton absorption in acid organelles and promotes the lysosomal escape of QDs.
\end{abstract}

\section{Introduction}

Cationic polymers with a large number of primary amine groups, such as polyamidoamine (PAMAM) dendrimer, polyethylenimine (PEI), and chitosan, are widely used in development of gene delivery carriers to promote cellular uptake via electrostatic interactions between positive and negative charges on the cell membrane [1-4]. Furthermore, these polymers are thought to have a strong $\mathrm{pH}$-buffering capacity that enhances proton absorption in acid organelles and osmotic pressure buildup across the organelle membrane. These processes in turn promote the endosomal escape and release of genes into the cytoplasm [5,6]. Quantum dots (QDs) are useful imaging tools for cellular labeling and monitoring due to their optical properties, such as a high fluorescence intensity, remarkable resistance to photobleaching, broad absorption spectra, and narrow emission spectra
$[7,8]$. Recently, QDs have been modified with PAMAM dendrimers for the efficient labeling of mesenchymal stem cells (MSCs). The uptake efficiency and cytosolic distribution of QDs in primary cultured MSCs are increased by modification of the PAMAM dendrimer [9]. However, it has not yet been investigated whether the chitosan monomer ( $D$ glucosamine) accelerates the uptake of modified QDs into cells.

The aim of this paper was to label osteoblastic cells by QDs for in vitro imaging. D-glucosamine hydrochloride is an extremely low molecular bioactive material with a potential buffering capacity. We synthesized carboxylated QDs to conjugate with $\mathrm{D}$-glucosamine in order to enhance both cellular uptake and endosomal escape in osteoblastic cells. The cellular uptake efficiency and intracellular distribution of D-glucosamine-conjugated QDs were investigated. 


\section{Materials and Methods}

2.1. Synthesis of CdSe Core Nanocrystals (NCs). Cadmium selenide (CdSe) NCs were synthesized and purified according to the method of Z. A. Peng and X. Peng [10]. Following purification, the vacuum-dried CdSe NCs were dissolved in chloroform and kept in the dark.

2.2. Carboxylation of QDs. Water-soluble CdSe core NCs were obtained using mercaptosuccinic acid as a surfacemodifying agent. Briefly, 20-30 mg of NCs was dissolved in chloroform, after which $3 \mathrm{~mL}$ of dimercaptosuccinic acid (Sigma, dissolved in $150 \mathrm{mM}$ PBS, pH 7.3) was added to $6 \mathrm{~mL}$ of NCs in chloroform. Water containing water-soluble CdSe NCs was carefully decanted and subjected to ultrafiltration to remove free (nonreacted) dimercaptosuccinic acid. The ultrafiltration was carried out using a concentrator (Vivaspin6/20, Sartorius AG, Gottingen, Germany).

2.3. Conjugation of Water-Soluble CdSe Core NCs with DGlucosamine. The water-soluble NCs were further conjugated with D-glucosamine. Carbodiimide chemistry was applied for conjugation, using $\mathrm{N}$-(3-dimethylaminopropyl)$\mathrm{N}^{\prime}$-ethylcarbodiimide hydrochloride (EDC) as a zero-length crosslinker. Briefly, $50 \mu \mathrm{L}$ of carboxylated QDs was mixed with $1 \mathrm{~mL}$ of D-glucosamine solution in PBS. Then, $100 \mu \mathrm{L}$ of $0.1 \mathrm{M}$ EDC stock solution in water was added to the mixture. Subsequently, the mixture reacted while stirring mildly for 24 hours at $4^{\circ} \mathrm{C}$.

2.4. Cell Culture and Cellular Uptake Studies. The osteoblastic cell line (NOS-1 cells [11]) from human osteosarcoma was purchased from Riken Cell Bank (Tsukuba city, Japan) and seeded in a $65 \mathrm{~mm}$ culture dish at a density of $1 \times 10^{5}$ cells in $\alpha$-MEM containing $10 \%$ fetal bovine serum and cultured in a humidified incubator at $37^{\circ} \mathrm{C}$ in an atmosphere of $5 \%$ $\mathrm{CO}_{2}$ and air. The subconfluent monolayer was passaged via trypsinization (trypsin-EDTA, Gibco Lab). The NOS-1 cells were seeded in a $35 \mathrm{~mm}$ glass-bottom culture dish (FluoroDish, World Precision Instruments, FL, USA) at a density of $6 \times 10^{5}$ cells for three, six, and 24 hours of incubation or a density of $3 \times 10^{5}$ cells for seven days of incubation. The final concentration of D-glucosamine conjugated with QDs was determined and adjusted to $0.05 \%$ in $\alpha$-MEM after preliminary experiments regarding cell growth and QD uptake were performed. In the cells cultured for seven days, normal $\alpha$-MEM was replaced three days after two days of incubation with conjugated QDs.

2.5. Fluorescent Staining of the Cell Membrane and Organelles. For cell membrane staining, fluorescent dye \{CellMask (C10045), Life Technologies, CA, USA $\}$ was used. After removing the culture medium from the dish, the dish was covered with $0.5 \mathrm{~mL}$ of staining solution $\{1 \mu \mathrm{L}$ of original solution per $1 \mathrm{~mL}$ of $\mathrm{PBS}(+)\}$ for five minutes in a humidified incubator at $37^{\circ} \mathrm{C}$. After removing the staining solution, the culture dish was rinsed with $\mathrm{PBS}(+)$ three times and covered with $\mathrm{PBS}(+)$ for observation. For cell organelle staining, fluorescent dye \{Organelle-ID RGB reagent I (ENZ53007), ENZO Life Sciences International, PA, USA $\}$, was used. After removing the culture medium from the dish, the dish was covered with $0.5 \mathrm{~mL}$ of staining solution $\{2 \mu \mathrm{L}$ of original solution per $1 \mathrm{~mL}$ of $\mathrm{PBS}(+)\}$ for 30 minutes in a humidified incubator at $37^{\circ} \mathrm{C}$. Subsequently, the culture dish was similarly washed and covered with PBS (+).

2.6. Confocal Laser Microscopy. Following cell membrane or organelle staining, the cells in $35 \mathrm{~mm}$ glass-bottom culture dishes were cultured in a $5 \% \mathrm{CO}_{2}$ incubator (H301-TC1HMTC, Okolab S.r.L., NA, Italy) for fluorescence observation then three-dimensionally analyzed (slicing width of cells: $1.5 \mu \mathrm{m}$ ) using a confocal laser microscope (TCS SL, Leica Microsystems GmbH, Wetzlar, Germany) to detect QDglucosamine conjugates delivered into the cells at different time intervals. The approximate fluorescence excitation/emission maxima for imaging of the QDs, cell membrane, and lysosomes were 385/525, 554/567, and 543/667, respectively.

\section{Results}

Over two hours, the stable observation of vital cells and fluorescence from QDs was possible using the 5\% $\mathrm{CO}_{2}$ incubator. As the yellow-green fluorescent brightness from QDs was extremely strong, it was easy to differentiate these molecules from other cellular elements. The cell membrane staining and confocal microscopic imaging easily provided observations of the QD uptake into the cells. After three hours of culture, QDs were observed inside the cells (Figure 1). After one day of culture, many QDs were taken up into the cells (Figure 2). After seven days of culture, the distribution of QDs was dramatically decreased inside the cells (Figure 3). After seven days of culture, the intranuclear distribution of QDs was not observed. After one day (Figure 4) and seven days (Figure 5) of culture in the control group (cells treated without conjugating with D-glucosamine), only a few QDs were observed. After merging the fluorescent images of the QDs and lysosomes, no overlap was observed between these two structures in the experimental group (Figure 2). However, partial overlap was detected in the images for the QDs and lysosomes in the control group (Figures 4 and 5).

\section{Discussion}

$\mathrm{D}$-glucosamine is used as an effective medicament in various fields of medicine and dentistry. For example, it is an attractive candidate for adjunctive therapy in patients with arthritis [12]. D-glucosamine also has a significant antipain effect in patients with osteoarthritis, a disease with low expectations of the value of treatment $[13,14]$. The present study documents another biological action of D-glucosamine, that is, the dramatic increase of the cellular uptake of QDs via attachment with the cell membrane due to a positive charge and the biocompatibility of conjugated D-glucosamine. This phenomenon was confirmed in control experiments, which clearly indicated that nonconjugated QDs have difficulty 


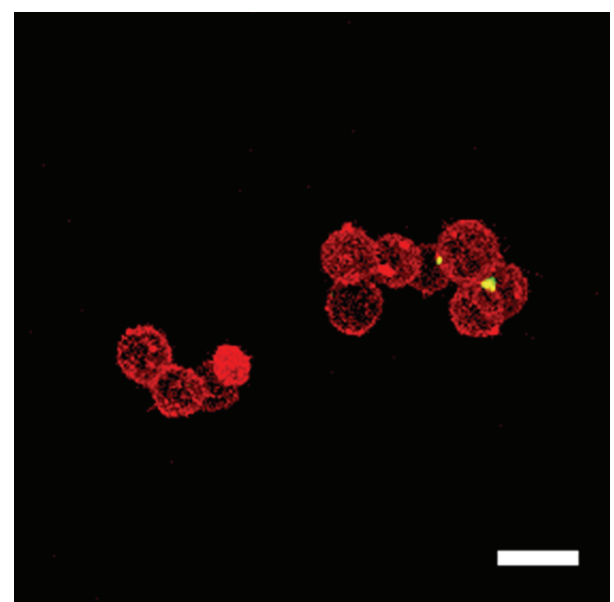

FIGURE 1: QD (high luminance of yellow-green fluorescence) inside the cell membrane (orange fluorescence) after three hours of culture. Scale bar $=15 \mu \mathrm{m}$.

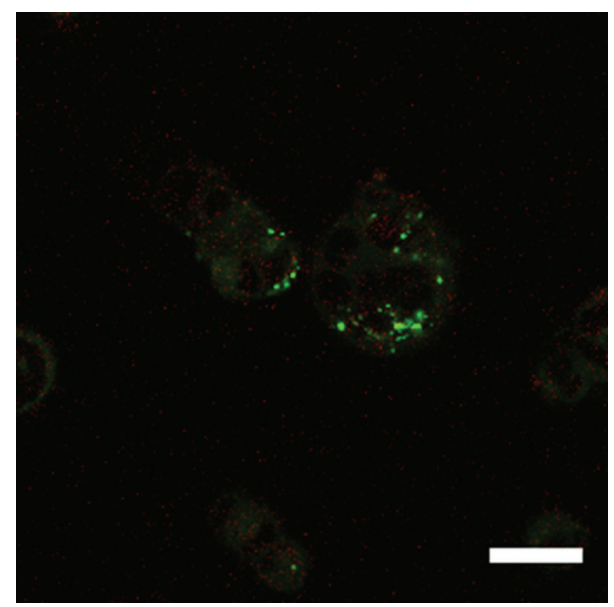

FIGURE 2: Many QDs (green fluorescence) are labeled inside the cells after one day of culture with D-glucosamine. Note the lack of overlap between the QDs and lysosomes (fluorescent red dots). Scale bar = $15 \mu \mathrm{m}$.

entering cells. Furthermore, additional interesting findings include the escape of QDs from lysosomes inside cells, as confirmed with the observation of merged fluorescence of both QDs and lysosomes. This is the first study to observe the stability and protection of intracellularly distributed QDs following the application of the monomer type of chitosan, $\mathrm{D}$-glucosamine. The proton sponge hypothesis, while not definitively proven, has been invoked to explain the relatively high transfection efficiency of other proton-spongetype materials, such as lipopolyamines [15, 16], PAMAM dendrimers [17], and various imidazole-containing polymers [18-20]. The original hypothesis proposed that polyethylenimine (PEI) buffering in lysosomes induced osmotic rupture and subsequent escape [21]. Although the proton sponge hypothesis based on the findings of a lack of lysosomal involvement is challenged in PEI-mediated gene transfer, a version of this hypothesis, whereby PEI buffering induces

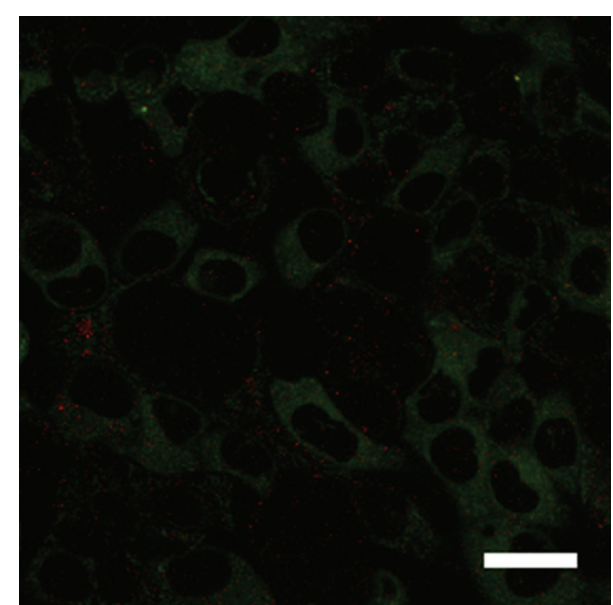

FIGURE 3: QD (green fluorescence) inside the cell after seven days of culture with D-glucosamine. Note the decrease in the number of QDs compared to that observed after one day of culture (Figure 2). Scale bar $=15 \mu \mathrm{m}$.

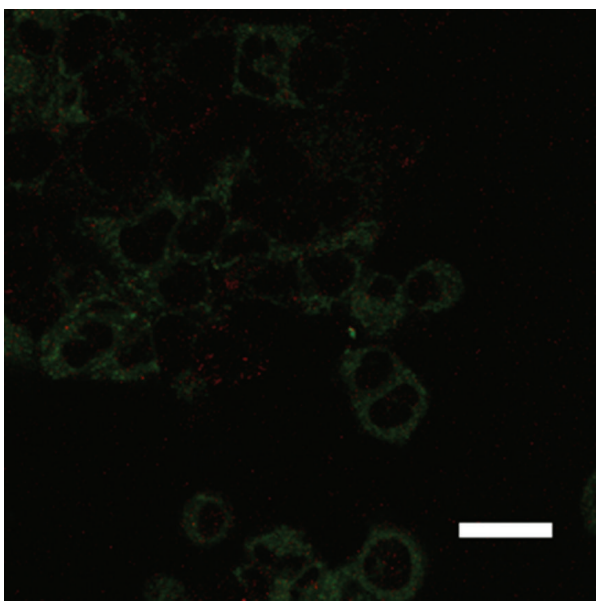

FIGURE 4: QD (green fluorescence) inside the cell after one day of culture without D-glucosamine. Note the partial overlap between the QDs and lysosomes (fluorescent red dots). Scale bar $=15 \mu \mathrm{m}$.

osmotic rupture in endosomes prior to fusion with lysosomes $[15,22]$, is consistent with the findings of Godbey et al. [23]. The $\mathrm{pH}$ of D-glucosamine hydrochloride is acidic (3.5-4.5). This acidic condition supports the proton sponge hypothesis involving escape from endosomes and lysosomes due to QD labeling for long periods. The new polycationic function of D-glucosamine (proton sponge hypothesis: escape from the degradative lysosomal trafficking pathway) is useful and meaningful for cell biology. This nanoimaging technology is therefore indispensable for investigating the distribution of bioactive materials, including applications in medical diagnosis. 


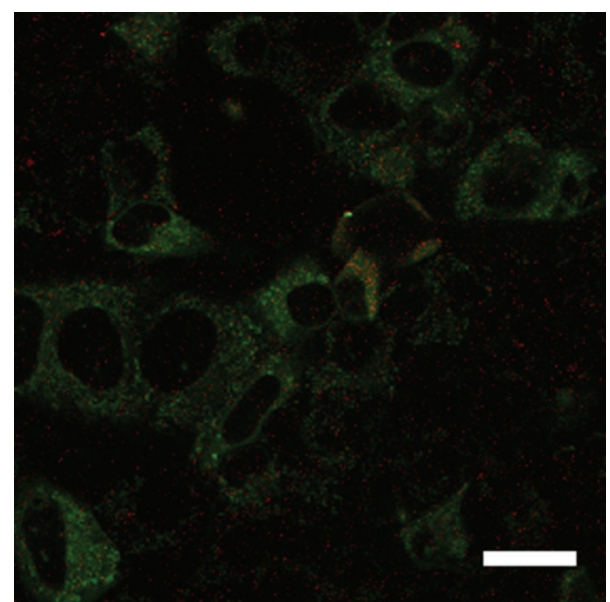

FIGURE 5: QD (green fluorescence) inside the cell after seven days of culture without D-glucosamine. Note the partial overlap between the QDs and lysosomes (fluorescent red dots). Scale bar $=15 \mu \mathrm{m}$.

\section{Conclusion}

This is the first study to investigate the uptake of carboxylated QDs conjugated with D-glucosamine into cultured osteoblastic cells. The interesting findings of this study include the escape of quantum dots from lysosomes in the cytoplasm, as confirmed with the merging of both QD fluorescence and specific fluorescent staining of lysosomes in the cytoplasm.

\section{Conflict of Interests}

The authors declare that there is no conflict of interests regarding the publication of this paper.

\section{Acknowledgment}

This work was supported by a Grant-in-Aid for Challenging Exploratory Research from the Japan Society, Contract Grant sponsor: Ministry of Education, Science, Sports and Culture of Japan, Contract Grant no. 22659345.

\section{References}

[1] Y. Higuchi, S. Kawakami, and M. Hashida, "Strategies for in vivo delivery of siRNAs: recent progress," BioDrugs, vol. 24, no. 3, pp. 195-205, 2010.

[2] S. Kawakami, Y. Higuchi, and M. Hashida, "Nonviral approaches for targeted delivery of plasmid DNA and oligonucleotide," Journal of Pharmaceutical Sciences, vol. 97, no. 2, pp. 726-745, 2008.

[3] S. C. W. Richardson, H. V. J. Kolbe, and R. Duncan, "Potential of low molecular mass chitosan as a DNA delivery system: biocompatibility, body distribution and ability to complex and protect DNA," International Journal of Pharmaceutics, vol. 178, no. 2, pp. 231-243, 1999.

[4] X. Liu, K. A. Howard, M. Dong et al., "The influence of polymeric properties on chitosan/siRNA nanoparticle formulation and gene silencing," Biomaterials, vol. 28, no. 6, pp. 1280-1288, 2007.
[5] A. Akinc, M. Thomas, A. M. Klibanov, and R. Langer, "Exploring polyethylenimine-mediated DNA transfection and the proton sponge hypothesis," Journal of Gene Medicine, vol. 7, no. 5, pp. 657-663, 2005.

[6] N. D. Sonawane, F. C. Szoka Jr., and A. S. Verkman, "Chloride Accumulation and Swelling in Endosomes Enhances DNA Transfer by Polyamine-DNA Polyplexes," The Journal of Biological Chemistry, vol. 278, no. 45, pp. 44826-44831, 2003.

[7] X. Michalet, F. F. Pinaud, L. A. Bentolila et al., "Quantum dots for live cells, in vivo imaging, and diagnostics," Science, vol. 307, no. 5709, pp. 538-544, 2005.

[8] I. L. Medintz, H. T. Uyeda, E. R. Goldman, and H. Mattoussi, "Quantum dot bioconjugates for imaging, labelling and sensing," Nature Materials, vol. 4, no. 6, pp. 435-446, 2005.

[9] Y. Higuchi, C. Wu, K.-L. Chang et al., "Polyamidoamine dendrimer-conjugated quantum dots for efficient labeling of primary cultured mesenchymal stem cells," Biomaterials, vol. 32, no. 28, pp. 6676-6682, 2011.

[10] Z. A. Peng and X. Peng, "Formation of high-quality CdTe, CdSe, and CdS nanocrystals using $\mathrm{CdO}$ as precursor," Journal of the American Chemical Society, vol. 123, no. 1, pp. 183-184, 2001.

[11] T. Hotta, T. Motoyama, and H. Watanabe, "Three human osteosarcoma cell lines exhibiting different phenotypic expressions," Acta Pathologica Japonica, vol. 42, no. 8, pp. 595-603, 1992.

[12] J. Hua, K. Sakamoto, and I. Nagaoka, "Inhibitory actions of glucosamine, a therapeutic agent for osteoarthritis, on the functions of neutrophils," Journal of Leukocyte Biology, vol. 71, no. 4, pp. 632-640, 2002.

[13] L. S. Lohmander and E. M. Roos, "Clinical update: treating osteoarthritis," The Lancet, vol. 370, no. 9605, pp. 2082-2084, 2007.

[14] J. S. Borer, H. Pouleur, E. Abadie et al., "Cardiovascular safety of drugs not intended for cardiovascular use: need for a new conceptual basis for assessment and approval," European Heart Journal, vol. 28, no. 15, pp. 1904-1909, 2007.

[15] J.-P. Behr, "The proton sponge: a trick to enter cells the viruses did not exploit," Chimia, vol. 51, no. 1-2, pp. 34-36, 1997.

[16] J.-S. Remy, C. Sirlin, P. Vierling, and J.-P. Behr, "Gene transfer with a series of lipophilic DNA-binding molecules," Bioconjugate Chemistry, vol. 5, no. 6, pp. 647-654, 1994.

[17] M. X. Tang and F. C. Szoka, "The influence of polymer structure on the interactions of cationic polymers with DNA and morphology of the resulting complexes," Gene Therapy, vol. 4, no. 8, pp. 823-832, 1997.

[18] D. W. Pack, D. Putnam, and R. Langer, "Design of imidazolecontaining endosomolytic biopolymers for gene delivery," Biotechnology and Bioengineering, vol. 67, pp. 217-223, 2000.

[19] P. Midoux and M. Monsigny, "Efficient gene transfer by histidylated polylysine/pDNA complexes," Bioconjugate Chemistry, vol. 10, no. 3, pp. 406-411, 1999.

[20] D. Putnam, C. A. Gentry, D. W. Pack, and R. Langer, "Polymerbased gene delivery with low cytotoxicity by a unique balance of side-chain termini," Proceedings of the National Academy of Sciences of the United States of America, vol. 98, no. 3, pp. 12001205, 2001.

[21] O. Boussif, F. LezoualC'H, M. A. Zanta et al., "A versatile vector for gene and oligonucleotide transfer into cells in culture and in vivo: polyethylenimine," Proceedings of the National Academy of Sciences of the United States of America, vol. 92, no. 16, pp. 72977301, 1995. 
[22] A. Akinc, M. Thomas, A. M. Klibanov, and R. Langer, "Exploring polyethylenimine-mediated DNA transfection and the proton sponge hypothesis," Journal of Gene Medicine, vol. 7, no. 5, pp. 657-663, 2005.

[23] W. T. Godbey, M. A. Barry, P. Saggau, K. K. Wu, and A. G. Mikos, "Poly(ethylenimine)-mediated transfection: a new paradigm for gene delivery," Journal of Biomedical Materials Research, vol. 51, pp. 321-328, 2000. 

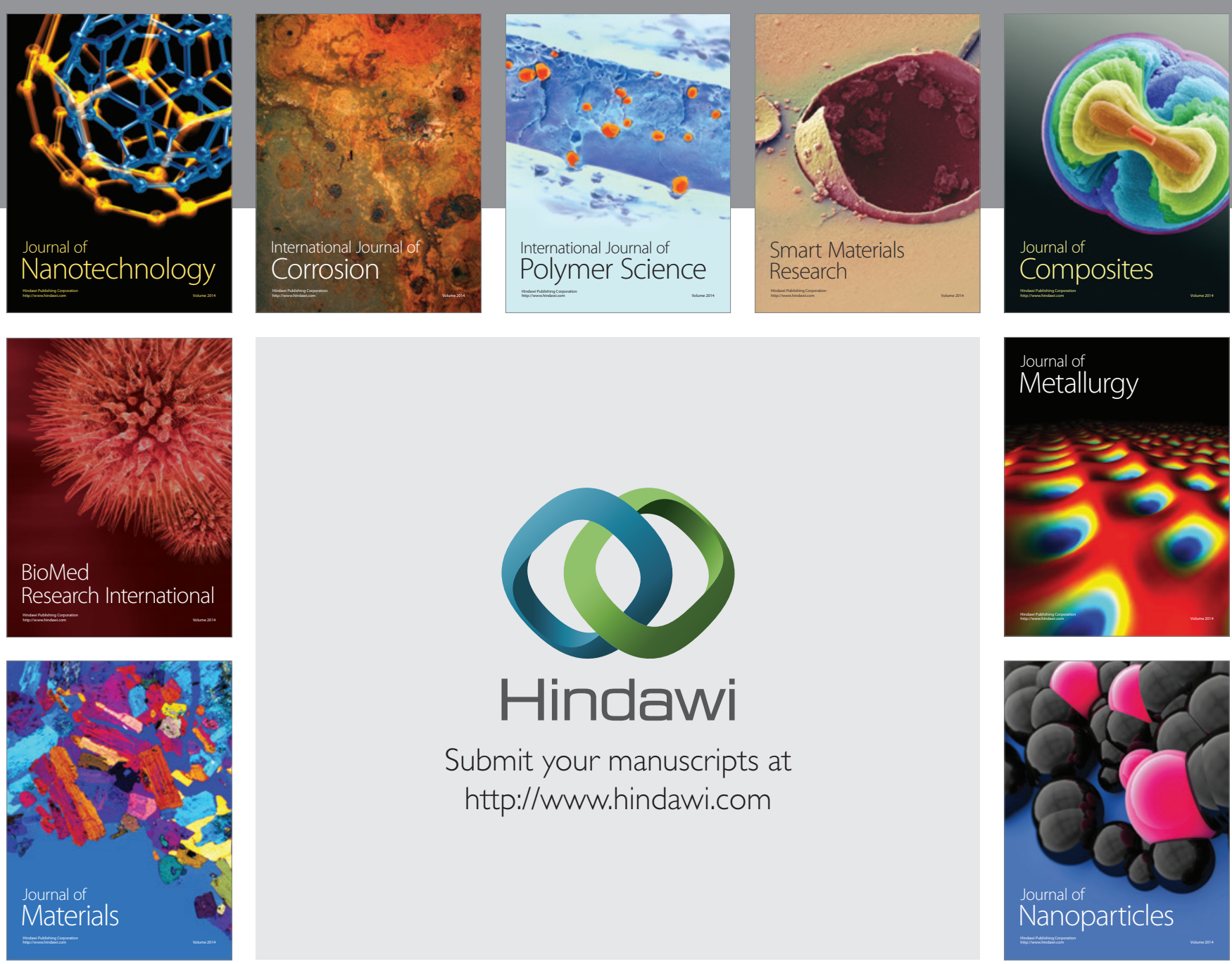

Submit your manuscripts at http://www.hindawi.com
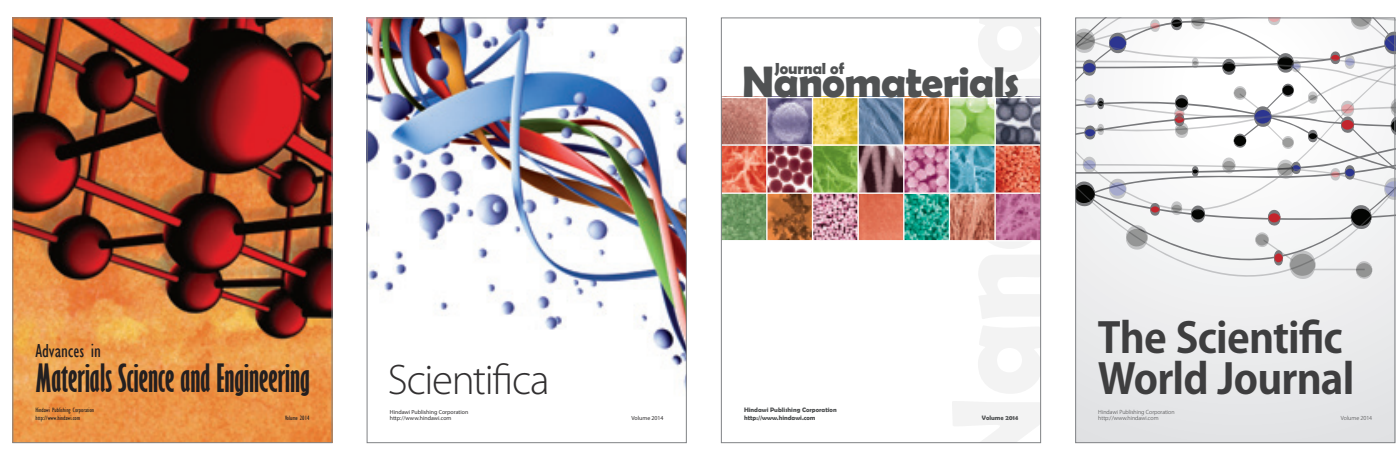

\section{The Scientific World Journal}
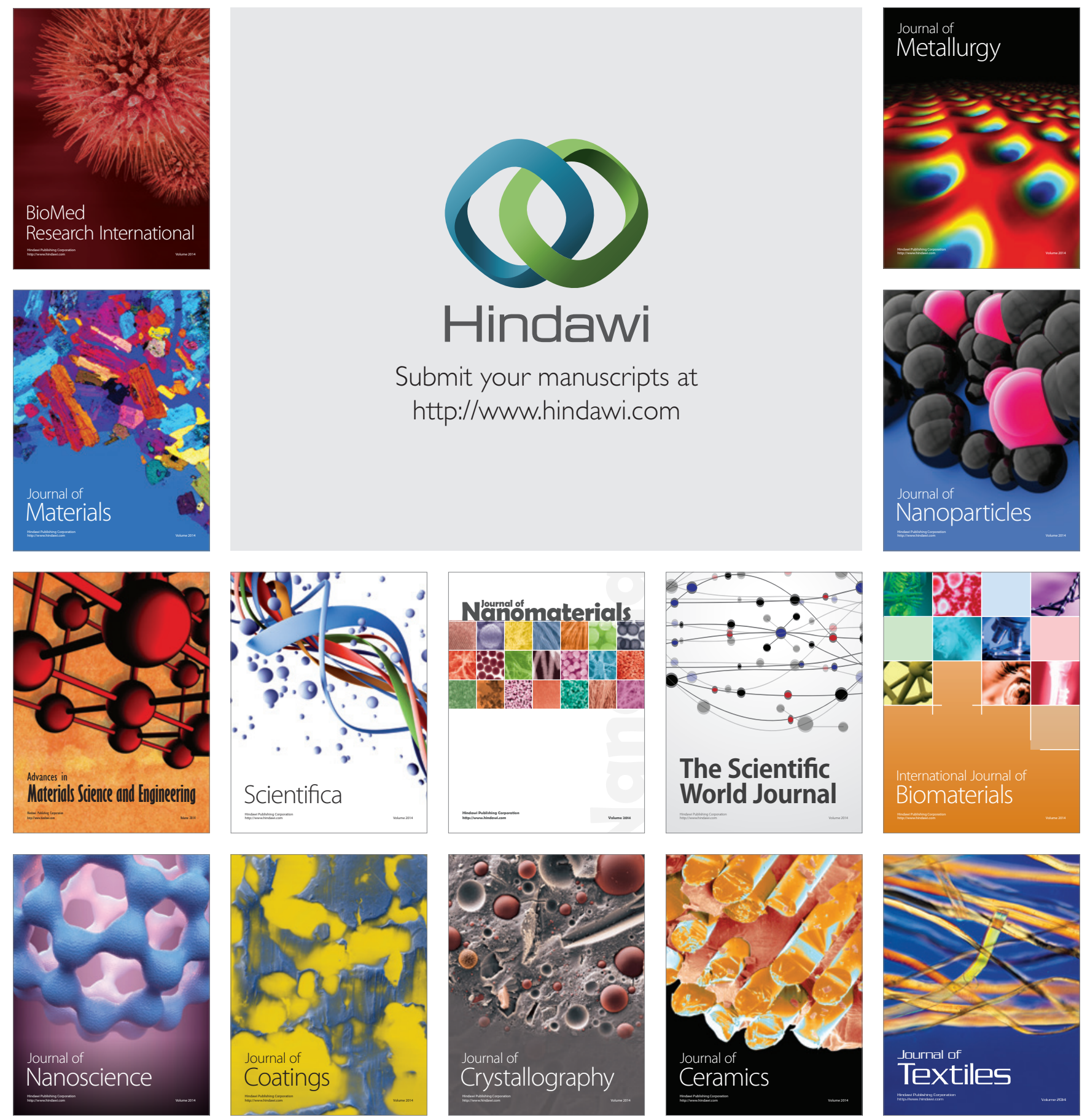